\title{
Different Cutoff Values for Increased Nuchal Translucency in First-Trimester Screening to Predict Fetal Chromosomal Abnormalities
}

\author{
Linjuan Su \\ Xiaoqing Wu $(\mathbb{D}$ \\ $\mathrm{Na}$ Lin \\ Xiaorui Xie \\ Meiying Cai (D) \\ Meiying Wang \\ Lin Zheng (iD) \\ Liangpu Xu
}

Fujian Provincial Key Laboratory for Prenatal Diagnosis and Birth Defect, Medical Genetic Diagnosis and Therapy Center, Fujian Provincial Maternity and Children's Hospital, Affiliated Hospital of Fujian Medical University, Fuzhou, Fujian, People's Republic of China
Correspondence: Liangpu Xu; Lin Zheng Fujian Provincial Key Laboratory for Prenatal Diagnosis and Birth Defect, Medical Genetic Diagnosis and Therapy Center, Fujian Provincial Maternity and Children's Hospital, Affiliated Hospital of Fujian Medical University, Fuzhou, Fujian,

People's Republic of China

Email ipxiu304@126.com;

zlinI536@I26.com
Introduction: Increased nuchal translucency (NT) is closely related to an increased risk of chromosomal abnormalities. However, the criterion of increased NT for invasive prenatal diagnosis remains controversial, as the cutoff values are inconsistent among countries. This study was conducted to compare the various cutoff values of increased NT and calculate the incidence of chromosomal abnormalities to determine the predictive ability of these cutoff values in conventional chromosome analysis.

Methods: A total of 3223 invasive samples with increased nuchal translucency (NT) or other non-ultrasound indications were collected from singleton pregnant women. Samples with isolated increased NT were divided into five groups based on the NT thickness: 909 samples in the NT $\geq 2.5 \mathrm{~mm}$ group, 819 samples in the NT $\geq 95$ th group, 547 samples in the NT $\geq 99$ th group, 527 samples in the NT $\geq 3.0 \mathrm{~mm}$ group, and 253 samples in the NT $\geq 3.5 \mathrm{~mm}$ group; 2301 samples with normal NT were considered as the control group. All five groups were karyotyped and the results were compared. The accuracy of the NT cutoff value for the screening of chromosomal abnormalities was assessed using receiver operating characteristic curve analysis.

Results: Detection of all chromosomal aberrations and trisomy 21 showed that the sensitivity and false-positive rate decreased sequentially in the NT $\geq 2.5 \mathrm{~mm}$, NT $\geq 95 \mathrm{th}$, NT $\geq 3 \mathrm{~mm}$, NT $\geq 99$ th, and NT $\geq 3.5 \mathrm{~mm}$ groups, whereas the specificity, positive predictive value, and false-negative rates increased sequentially. Comprehensive analysis of various factors, including sensitivity and specificity, revealed values equal to or higher than the calculated 95th percentile of NT distribution, which showed a sensitivity of $49.2 \%$ and specificity of $75.67 \%$ for detecting all aneuploidies and a sensitivity of $64 \%$ and specificity of $75.45 \%$ for trisomy 21 , exhibiting the highest ability for the screening of chromosomal defects in first-trimester screening.

Conclusion: For different thresholds of NT thickness, values equal to or higher than the calculated 95th percentile of the NT distribution showed the highest ability for the screening of chromosomal defects in first-trimester screening.

Keywords: nuchal translucency, cutoff value, invasive prenatal diagnosis, chromosomal abnormalities, first-trimester screening, crown-rump length, trisomy 21

\section{Introduction}

Nuchal translucency (NT) refers to the transient subcutaneous collection of fluids present behind the fetal neck and can be detected using ultrasonography at 11-14 weeks of gestation as an important ultrasonic parameter during the first-trimester of pregnancy. ${ }^{1}$ An increased NT thickness is associated with adverse pregnancy outcomes, including a higher risk of chromosomal and structural abnormalities. ${ }^{2,3}$ 
Invasive prenatal diagnostic techniques can be used to exclude chromosome anomalies when an abnormal fetal NT thickness is detected. However, the cutoff value of increased NT thickness in invasive prenatal diagnosis is controversial and differs among countries. Some countries use fixed cutoff values such as $\geq 3.5$, $\geq 3.0$, or $\geq 2.5 \mathrm{~mm}$ with a 45-84 mm crown-rump length (CRL), as detected by karyotyping or CMA. ${ }^{1,4}$ Other countries utilize cutoff values equal to or higher than either the calculated 95th ( $\geq 95$ th) or 99 th $(\geq 99$ th) percentile of the NT distribution at 45-84 mm CRL. ${ }^{5-7}$ Inconsistent standards result in inaccurate data analysis, affecting the potential use of NT in prenatal diagnosis. This study was conducted to compare various cutoff values of NT, calculate the incidence of chromosomal aberrations, and assess the predictive ability of NT in prenatal diagnosis and first-trimester screening.

\section{Materials and Methods Ethical Compliance}

All procedures followed in this study were performed in accordance with the ethical standards mentioned in the Declaration of Helsinki and Methods for Ethical Review of Biomedical Research involving People by the State and the Ethics Committee of Fujian Provincial Maternity and Children's Hospital (ethics approval number 2016-051). Written informed consent was obtained from all participants.

\section{Study Population}

In this retrospective cohort study, 3223 invasive samples were collected from singleton pregnant women admitted to Fujian Provincial Maternity and Children's Hospital, China, between October 2008 and December 2020. Transabdominal ultrasound examination was performed to diagnose any major fetal defects in the first trimester. This examination included evaluation of fetal NT thickness and the following secondary ultrasound markers of chromosomal abnormalities: absence or dysplasia of the nasal bone, reversal or absence of a-wave in ductus venosus, and tricuspid regurgitation in tricuspid valve flow. The NT of infants was measured at a CRL of 45$84 \mathrm{~mm}$, and gestational age was determined using CRL during the 11-14-week scan. ${ }^{8}$ Specimens with increased NT were divided into five groups based on the NT thickness: $\mathrm{NT} \geq 2.5 \mathrm{~mm}$ (group A), NT $\geq 95$ th percentile (group B), NT $\geq 99$ th percentile (group C), NT $\geq 3.0 \mathrm{~mm}$ (group D), and NT $\geq 3.5 \mathrm{~mm}$ (group E). The karyotyping results of the five groups were compared. Cases with other ultrasound abnormalities presented during the 1114-week scan were excluded, whereas those with structural malformation were not excluded during secondtrimester screening. A total of 2301 fetuses with NT less than $2.5 \mathrm{~mm}$ or 95 th who underwent routine cytogenetic testing for non - ultrasound indications, such as advanced age, abnormal delivery, positive serum screening and the thalassemia gene mutation, constituted the control group.

\section{Statistical Analysis}

Maternal clinical characteristics and experimental results were analyzed using the SPSS statistical software. Data are presented as means. One-way analysis of variance and Chi-square test were performed to determine the significance of differences between groups. A p-value of less than 0.05 was considered to indicate statistically significant results. The screening accuracy of the threshold was assessed using receiver operating characteristic (ROC) curve analysis for chromosomal abnormalities. All calculations were performed using SPSS 18 software (SPSS, Inc., Chicago, IL, USA).

\section{Results}

Among the 3223 pregnant women evaluated, 8 participants without increased NT and with failed cell cultures were excluded. Karyotyping of 3215 samples was performed and all of them met the inclusion criteria. The 914 fetuses with increased NT were classified into five groups: group A (NT $\geq 2.5 \mathrm{~mm})$ included 909 (99.45\%) fetuses, group B (NT $\geq 95$ th) included 819 (89.61\%), group C (NT $\geq 99$ th) included 547 (59.85\%), group D (NT $\geq 3 \mathrm{~mm})$ included $527(57.65 \%)$, and group E (NT $\geq 3.5 \mathrm{~mm})$ included 253 $(22.54 \%)$ fetuses. The clinical characteristics of the study population are shown in Table 1. Except for significant differences in the NT thickness, there were no differences in maternal age, parity, and sex ratio among the groups based on one-way analysis of variance and Chi-square test.

We identified 153 chromosomal abnormalities (5.29\%) in 73 cases in the control group and 80 cases in the five groups with increased NT (Appendix S1). The numbers of abnormal karyotypes detected in groups A-E were 78 (78/909, 8.58\%), $75(75 / 819,9.16 \%), 58(58 / 547,10.60 \%), 59(59 / 527$, $11.2 \%)$, and $41(41 / 253,16.21 \%)$, respectively. In the detection of all aneuploidies, the sensitivity and false-positive rate decreased from groups A to E. For specificity, the positive predictive value and false-negative rates increased 
Table I Clinical Characteristics of the Study Population

\begin{tabular}{|c|c|c|c|c|c|}
\hline & Group A & Group B & Group C & Group D & Group E \\
\hline & $\mathrm{NT} \geq 2.5 \mathrm{~mm}$ & NT $\geq 95$ th & NT $\geq 99$ th & $\mathbf{N T} \geq \mathbf{3} \mathbf{m m}$ & NT $\geq 3.5 \mathrm{~mm}$ \\
\hline & $\mathrm{n}=909$ & $\mathrm{n}=819$ & $\mathrm{n}=547$ & $\mathrm{n}=527$ & $n=253$ \\
\hline Maternal age (year) $(\text { mean } \pm S D)^{a}$ & $30.9 \pm 4.587$ & $30.8 \pm 4.536$ & $30.49 \pm 4.486$ & $30.51 \pm 4.481$ & $30.58 \pm 4.388$ \\
\hline $\mathrm{NT}(\mathrm{mm})(\text { mean } \pm \mathrm{SD})^{\mathrm{b}}$ & $3.31 \pm 0.951$ & $3.39 \pm 0.9966$ & $3.71 \pm 1.038$ & $3.76 \pm 1.033$ & $4.42 \pm 1.166$ \\
\hline Crown-rump length $(\mathrm{CRL})(\mathrm{mm})(\text { mean } \pm \mathrm{SD})^{\mathrm{a}}$ & $66 \pm 8.756$ & $65.2 \pm 8.891$ & $65.07 \pm 8.522$ & $65.79 \pm 9.102$ & $65.04 \pm 9.181$ \\
\hline $\operatorname{Parity}(\mathrm{n})(\text { mean } \pm S D)^{\mathrm{a}}$ & $2.37 \pm 1.260$ & $2.36 \pm 1.257$ & $2.31 \pm 1.224$ & $2.32 \pm 1.220$ & $2.27 \pm 1.238$ \\
\hline Female fetuses $(n(\%))^{c}$ & $310(34.10)$ & $28 I(34.3 I)$ & $202(36.93)$ & $186(35.29)$ & $95(37.55)$ \\
\hline
\end{tabular}

Notes: ${ }^{a}$ Group $A$ vs group $B$ vs group $C$ vs group $D$ and vs group $E ; P>0.05$. ${ }^{b} G$ roup $A$ vs group $B ; P>0.05$, group $C$ vs group $D ; P>0.05$, group $A$ vs group $C$ vs group $D$ and group $E ; P<0.05$, group $C$ vs group $A$ vs group $B$ and group $E ; P<0.05$. ${ }^{c}$ Chi-square tests; $P>0.05$.

sequentially from groups A to $\mathrm{E}$ (Table 2). NT $\geq 2.5 \mathrm{~mm}$ showed the highest sensitivity (50.98\%), highest falsepositive rate $(27.17 \%)$, and lowest specificity $(72.83 \%)$. The highest specificity was found for the fixed NT cut-off of $3.5 \mathrm{~mm}$, at which the false-negative rate reached the highest value of $73.2 \%$ and lowest sensitivity of $26.80 \%$ (Table 2). The ROC curves used to determine the accuracy of different cut-off values in NT screening for aneuploidy are shown in Figure 1.

In each group, trisomy 21 was the most common abnormality detected (Table 3 ). Seventy-five cases of trisomy 21 were found, including $25(25 / 75,33.33 \%)$ in the normal NT group and $50(50 / 75,66.67 \%)$ in the increased NT group. Among pregnancies with an NT-value of $\geq 2.5 \mathrm{~mm}, 49(49 / 75,65.33 \%)$ cases of trisomy 21 were detected, with 48 (48/75, 64.00\%), 39 (39/75, 52.00\%), 38 $(38 / 75,50.67 \%)$, and $26(26 / 75,34.67 \%)$ were detected in the NT $\geq 95 \mathrm{th}, \mathrm{NT} \geq 3 \mathrm{~mm}, \mathrm{NT} \geq 99 \mathrm{th}$, and NT $\geq 3.5 \mathrm{~mm}$ groups, respectively. The optimum effectiveness of different cut-off values in NT screening for trisomy 21 was assessed using ROC curve analysis as shown in Figure 2.

\section{Discussion}

\section{Criteria for Increased NT}

The use of fetal NT was first described in 1992 as an ultrasound screening marker for identifying chromosomal defects in the first-trimester of pregnancy. ${ }^{9}$ Most subsequent studies confirmed that increased NT thickness is related to chromosomal abnormalities, most commonly aneuploidy. ${ }^{2,3}$ To detect chromosomal abnormalities, invasive prenatal diagnosis is required.

Most countries choose to adopt a different percentile for the NT distribution or absolute values as a threshold for estimating whether NT has increased, leading to ambiguity in the NT threshold value (Table 4). The calculated 95th
( $\geq 95$ th) or 99th ( $\geq 99$ th) percentile of the NT distribution varies according to the length of the CRL; this value increases with an increase in the CRL length. The absolute NT value indicating a risk of abnormal karyotypes in the fetus at CRL of 45$84 \mathrm{~mm}$ does not depend on gestational age or physiological changes. The multiple standards for NT cutoff values have led to ambiguity in the sensitivity and specificity, resulting in some birth defects remaining undetected and the waste of medical resources. Compared with the economic cost of prenatal diagnosis, the birth of a fetus with a chromosomal abnormality has a burden on the family, indicating a more appropriate cutoff value with higher detection efficiency should be determined.

\section{Screening Efficiency of Different NT Thresholds}

In this study, the incidence rates of chromosomal abnormalities under different threshold values of NT were $8.58 \%$, $9.16 \%, 10.60 \%, 11.20 \%$, and $16.21 \%$ in groups $\mathrm{A}-$ E. A higher critical value of NT was associated with a higher incidence of aberrant chromosomes, confirming that the degree of fetal NT thickening is positively correlated with the risk of chromosomal abnormalities. To avoid the effect of other ultrasound indicators, we excluded fetuses with additional abnormal ultrasound structures and other ultrasonographic soft markers in the first-trimester; only fetuses with increased NT were included in the study. Moreover, chromosomal polymorphism and balanced translocations inherited from the parents were classified as normal karyotypes in the experimental results; therefore, the detection rate of abnormal karyotypes for each threshold value in this study is lower than that in other reports. ${ }^{4,10-12}$

A higher cutoff value of NT led to a higher specificity of abnormal karyotypes. At an $\mathrm{NT} \geq 3.5 \mathrm{~mm}$, the 


\begin{tabular}{|c|c|c|c|}
\hline \multicolumn{2}{|c|}{ 站 ฮ̊ } & & 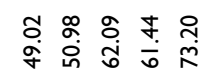 \\
\hline \multicolumn{2}{|c|}{$\frac{a}{a} \stackrel{0}{\varrho}$} & 1 & 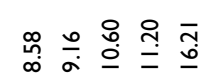 \\
\hline \multicolumn{2}{|c|}{$\frac{\alpha}{2} \stackrel{0}{d}$} & 1 & 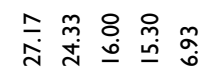 \\
\hline \multicolumn{2}{|c|}{ 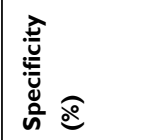 } & 1 & 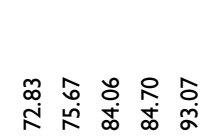 \\
\hline \multicolumn{2}{|c|}{ 童 } & 1 & 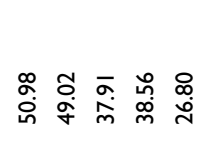 \\
\hline \multirow{6}{*}{ 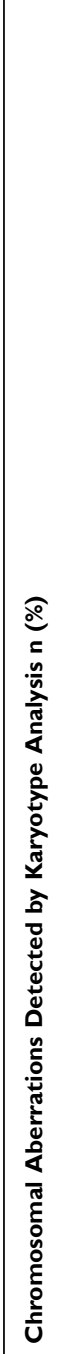 } & 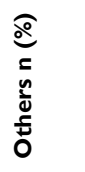 & 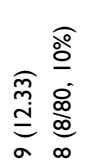 & 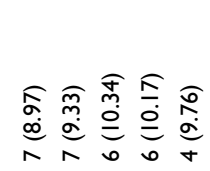 \\
\hline & 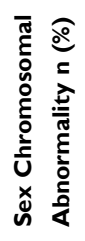 & 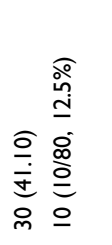 & 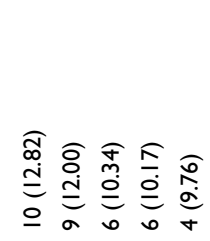 \\
\hline & 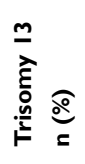 & 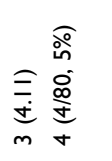 & 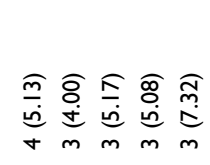 \\
\hline & 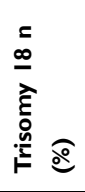 & 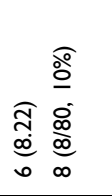 & 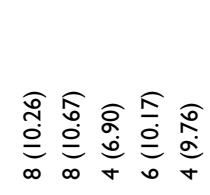 \\
\hline & 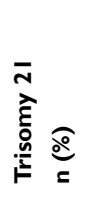 & 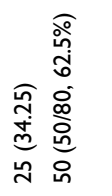 & 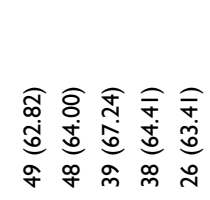 \\
\hline & 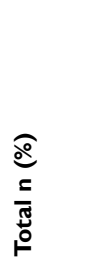 & 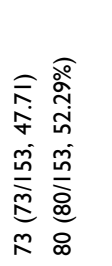 & 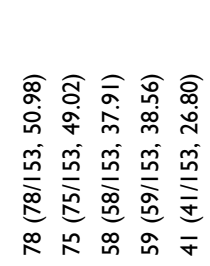 \\
\hline \multicolumn{2}{|l|}{$\begin{array}{l}\text { है } \\
\text { z }\end{array}$} & \multicolumn{2}{|c|}{ 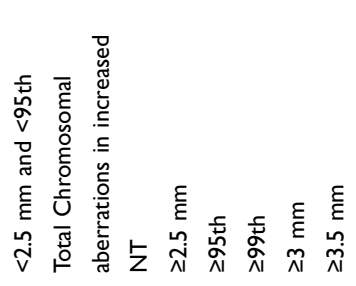 } \\
\hline
\end{tabular}

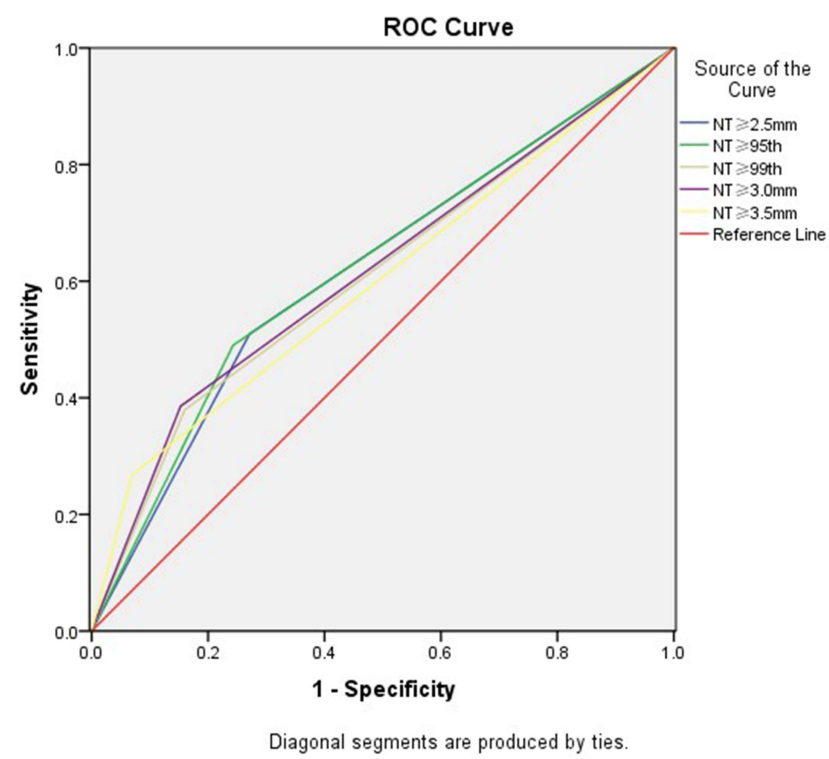

Figure I Effectiveness of screening for chromosomal aberrations using different cutoff values for fetal nuchal translucency (NT). ROC curve of NT: NT $\geq 2.5 \mathrm{~mm}$ : area under the curve $(\mathrm{AUC})=0.619,95 \%$ confidence interval $(\mathrm{Cl})=0.57 \mathrm{I}-0.667$, $\mathrm{P}<0.000 \mathrm{I}$. NT $\geq 95$ th: AUC $=0.624,95 \% \mathrm{Cl}=0.575-0.672, \mathrm{P}<0.000 \mathrm{I}$. NT $\geq 99$ th: $\mathrm{AUC}=0.610,95 \% \mathrm{Cl}=0.560-0.660, \mathrm{P}<0.0001 . \mathrm{NT} \geq 3.0 \mathrm{~mm}: \mathrm{AUC}=0.616,95 \%$ $\mathrm{Cl}=0.566-0.666, \mathrm{P}<0.000 \mathrm{I} . \mathrm{NT} \geq 3.5 \mathrm{~mm}: \mathrm{AUC}=0.599,95 \% \mathrm{Cl}=0.548-0.65 \mathrm{I}$, $P<0.0001$.

specificity of total chromosomal aberrations reached $93.07 \%$ and relative sensitivity decreased to only $26.80 \%$, indicating that chromosomal abnormalities were undetectable in many fetuses. Because of the harmfulness of chromosomal abnormalities, we tested the falsenegative rate, which also increased with increasing NT critical values. Compared with the threshold of NT $\geq 2.5 \mathrm{~mm}$ which showed high sensitivity, 24 cases of trisomy 21, 4 cases of trisomy 18, 1 case of trisomy 13, and 6 cases of sex chromosome abnormality were undetectable at $\mathrm{NT} \geq 3.5 \mathrm{~mm}$.

To determine the screening efficiency, we assessed whether diverse NT threshold values could be used to predict chromosomal defects using ROC curve analysis. ROC is a comprehensive index reflecting the sensitivity and specificity of continuous variables, which can be used to explore the accuracy of an experiment. The area under the ROC curve can accurately reflect the authenticity of the screening experiment. Typically, a larger area under the curve (AUC) indicates a higher screening efficiency. For all chromosomal aberrations, the ROC curve indicated that the highest comprehensive detectability was observed at a cutoff value of more than 95th NT and AUC of 0.624 (95\% CI: 0.575-0.672). Among common aneuploidy chromosomal abnormalities, the incidence of trisomy 18 and 
Table 3 Performance of NT for Detection of Trisomy 21

\begin{tabular}{|l|l|l|l|l|l|l|}
\hline \multirow{2}{*}{ NT (mm) } & $\begin{array}{l}\text { Trisomy 2I Detected by } \\
\text { Karyotype Analysis n (\%) }\end{array}$ & Sensitivity (\%) & Specificity (\%) & FPR (\%) & PPV (\%) & FNR (\%) \\
\cline { 2 - 6 } & Trisomy 2I n (\%) & & & & \\
\hline$<2.5 \mathrm{~mm}$ or <95th & $25(25 / 75,33.33)$ & - & - & - & - & - \\
$\geq 2.5 \mathrm{~mm}$ & $49(49 / 75,65.33)$ & 65.33 & 72.61 & 27.39 & 5.39 & 34.67 \\
$\geq 95 \mathrm{th}$ & $48(48 / 75,64.00)$ & 64.00 & 75.45 & 24.55 & 5.86 & 36.00 \\
$\geq 99 \mathrm{th}$ & $39(39 / 75,52.00)$ & 52.00 & 83.82 & 16.18 & 7.13 & 48.00 \\
$\geq 3 \mathrm{~mm}$ & $38(38 / 75,50.67)$ & 50.67 & 84.43 & 15.57 & 7.21 & 49.33 \\
$\geq 3.5 \mathrm{~mm}$ & $26(26 / 75,34.67)$ & 34.67 & 92.77 & 7.23 & 10.28 & 65.33 \\
\hline
\end{tabular}

Abbreviations: NT, nuchal translucency; FPR, false-positive rate; PPV, positive predictive value.

13 is less than that of trisomy 21 , and the fetus typically exhibits severe multiple malformations in the uterus, along with a poor prognosis and short survival. The incidence of trisomy 21 is quite high. Some fetuses are not accompanied by obvious multiple malformations before birth and survive for a long time, leading to greater burden on the family and society. Therefore, screening for trisomy 21 is particularly important. For trisomy 21 , the comprehensive detectability of NT $\geq 95$ th was highest, with an AUC of 0.700 (95\% CI: $0.637-0.755)$.

\section{NT and Other Screening Methods}

As an indicator in the first-trimester, NT is closely related to the risk of fetal chromosomal abnormalities; however as

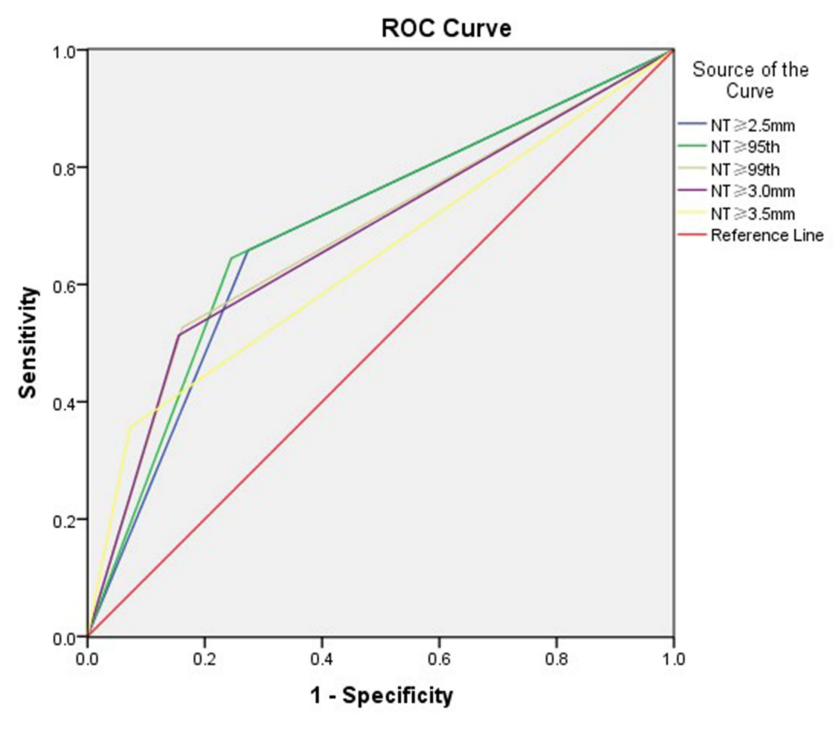

Diagonal segments are produced by ties.

Figure 2 Effectiveness of screening for trisomy 21 using diffident cutoff values of fetal nuchal translucency (NT). ROC curve of NT: NT $\geq 2.5 \mathrm{~mm}$ : area under the curve $(\mathrm{AUC})=0.692,95 \%$ confidence interval $(\mathrm{Cl})=0.630-0.755, \mathrm{P}<0.000 \mathrm{I}$. NT $\geq 95$ th: $A U C=0.700,95 \% \mathrm{Cl}=0.637-0.755, P<0.000 \mathrm{I}$. NT $\geq 99$ th: $A U C=0.682$, $95 \% \mathrm{Cl}=0.614-0.75 \mathrm{I}, \mathrm{P}<0.000 \mathrm{I}$. NT $\geq 3.0 \mathrm{~mm}$ : AUC $=0.679,95 \% \mathrm{Cl}=0.610$ $0.748, \mathrm{P}<0.000 \mathrm{I}$. NT $\geq 3.5 \mathrm{~mm}: \mathrm{AUC}=0.642,95 \% \mathrm{Cl}=0.569-0.714, \mathrm{P}<0.0001$.
Table 4 Criteria of Increased NT in Different Countries

\begin{tabular}{|l|l|}
\hline Country & Cutoff Value of NT \\
\hline Finland & $95 \mathrm{th}^{17}$ \\
UK & $3.5 \mathrm{~mm}^{4}, 3.0 \mathrm{~mm}^{10}$ \\
Israel & $99 \mathrm{th}^{11}$ \\
Netherlands & $95 \mathrm{th}^{12}$ \\
Spain & $99 \mathrm{th}^{7}{ }^{72.5 \mathrm{~mm}^{18}}$ \\
Germany & $95 \mathrm{th}^{19} 3.5 \mathrm{~mm}^{20}$ \\
USA & $3 \mathrm{~mm}^{21} 95 \mathrm{th}^{22}$ \\
China & $3.5 \mathrm{~mm}^{23}, 3.0 \mathrm{~mm}^{24}$ \\
Switzerland & $95 \mathrm{th}^{25}$ \\
Romania. & $3.5 \mathrm{~mm}^{26}$ \\
France & $3.5 \mathrm{~mm}^{27}$ \\
Australia & $3.5 \mathrm{~mm}^{28}$ \\
Sweden & $3.5 \mathrm{~mm}^{29}$ \\
Turkey & $3.0 \mathrm{~mm}^{30}$ \\
\hline
\end{tabular}

a single indicator, its screening ability is limited. Secondary ultrasound markers evaluated during prenatal screening, such as nasal bone status, tricuspid regurgitation flow, ductus venosus flow, increased the detection rate of trisomy 21 and decreased false-positive rate; together first trimester serum biochemistry analyses, chromosomal abnormalities including trisomy 21 can be detected in up to $100 \%$ of cases. ${ }^{13,14} \mathrm{NT}$ can also indicate the risk of fetal chromosomal microdeletion and microduplication, ${ }^{15}$ and an additional $8.2 \%$ of cases with copy number variation can be detected using chromosomal microarray analysis. ${ }^{16}$

\section{Conclusions}

In conclusion, an increased NT thickness indicates an increased risk of chromosome abnormalities in fetuses. NT assessment should be performed during the firsttrimester. Comparison of different cutoff values for increased NT in a defined cohort of pregnancies showed 
that the NT $\geq 95$ th percentile had the highest screening ability for chromosomal defects and should be adopted as a threshold for increased NT during screening in the first-trimester.

\section{Acknowledgments}

We thank all the patients and research workers for their participation.

\section{Disclosure}

The authors declare no conflicts of interest.

\section{References}

1. Giorlandino C, Cignini P, Padula F, et al. Effects of exogenous progesterone on fetal nuchal translucency: an observational prospective study. Am J Obstet Gynecol. 2015;212:335.e1-e7. doi:10.1016/j. ajog.2014.10.003

2. Baer RJ, Norton ME, Shaw GM, et al. Risk of selected structural abnormalities in infants after increased nuchal translucency measurement. Am J Obstet Gynecol. 2014;211:675 e1-19. doi:10.1016/j.ajog.2014.06.025

3. Kagan KO, Avgidou K, Molina FS, et al. Relation between increased fetal nuchal translucency thickness and chromosomal defects. Obstet Gynecol. 2006;107:6-10. doi:10.1097/01. AOG.0000191301.63871.c6

4. Syngelaki A, Guerra L, Ceccacci I, et al. Impact of holoprosencephaly, exomphalos, megacystis and increased nuchal translucency on first-trimester screening for chromosomal abnormalities. Ultrasound Obstet Gynecol. 2017;50:45-48. doi:10.1002/uog.17286

5. Wulff CB, Gerds TA, Rode L, et al. The risk of fetal loss associated with invasive testing following combined first trimester risk screening for Down syndrome - a national cohort of 147987 singleton pregnancies. Ultrasound Obstetr Gynecol. 2016;47:38-44. doi:10.1002/uog. 15820

6. Hellmuth SG, Pedersen LH, Miltoft CB, et al. Increased nuchal translucency thickness and risk of neurodevelopmental disorders. Ultrasound Obstetr Gynecol. 2017;49:592-598. doi:10.1002/ uog. 15961

7. Miranda J, Paz YMF, Borobio V, et al. Can cell-free DNA testing be used in pregnancies with increased nuchal translucency? Ultrasound Obstet Gynecol. 2019;34(4):624-628.

8. Hadlock FP, Shah YP, Kanon DJ, et al. Fetal crown-rump length: reevaluation of relation to menstrual age (5-18 weeks) with highresolution real-time US. Radiology. 1992;182:501-505. doi:10.1148/ radiology.182.2.1732970

9. Nicolaides KH, Azar G, Byrne D, et al. Fetal nuchal translucency: ultrasound screening for chromosomal defects in first trimester of pregnancy. BMJ. 1992;304:867. doi:10.1136/bmj.304.6831.867

10. Khalil A, Mahmoodian N, Kulkarni A, et al. Estimation of detection rates of aneuploidy in high-risk pregnancy using an approach based on nuchal translucency and non-invasive prenatal testing: a cohort study. Fetal Diagn Ther. 2015;38:254-261. doi:10.1159/000381182

11. Yaron Z, Roni Z, Zvi K. The added value of detailed early anomaly scan in fetuses with increased nuchal translucency. Prenat Diagn. 2017;37:235-243. doi:10.1002/pd.4997

12. Bardi F, Bosschieter P, Verheij J, et al. Is there still a role for nuchal translucency measurement in the changing paradigm of first trimester screening? Prenat Diagn. 2019;40:197-205. doi:10.1002/pd.5590

13. Sivanathan J, Thilaganathan B. Book: genetics for obstetricians and gynaecologists: chapter:Genetic markers on ultrasound scan. Best Pract Res Clin Obstet Gynaecol. 2017;42:64-85.
14. Hsiao CH, Cheng PJ, Shaw S, et al. Extended first-trimester screening using multiple sonographic markers and maternal serum biochemistry: a five-year prospective study. Fetal Diagn Ther. 2014;35:296-301. doi:10.1159/000357564

15. Su L, Huang H, An G, et al. Clinical application of chromosomal microarray analysis in fetuses with increased nuchal translucency and normal karyotype. Mol Genet Genom Med. 2019;7:e811. doi:10.1002/mgg3.811

16. Sinajon P, Chitayat D, Roifman M, et al. Microarray and RASopathy-disorder testing in fetuses with increased nuchal translucency. Ultrasound Obstet Gynecol. 2020;55:383-390. doi:10.1002/uog.20352

17. Yräs $\mathrm{O}$, Eronen $\mathrm{M}$, Tikkanen $\mathrm{M}$, et al. Long-term neurodevelopmental outcome of children from euploid pregnancies with increased nuchal translucency in the first trimester screening. Prenat Diagn. 2015;35:362-369. doi:10.1002/pd.4548

18. De La Paz-gallardo MJ, García FSM, De Haro-muñoz T, et al. Quantitative-fluorescent-PCR versus full karyotyping in prenatal diagnosis of common chromosome aneuploidies in southern Spain. Clin Chem Lab Med. 2015;53:1333-1338. doi:10.1515/cclm-2014-0781

19. Meyberg-Solomayer G, Hamza A, Takacs Z, et al. The significance of anterolateral neck cysts in early diagnosis of fetal malformations. Prenat Diagn. 2016;36:332-337. doi:10.1002/pd.4785

20. Kagan KO, Sonek J, Sroka A, et al. False-positive rates in screening for trisomies 18 and 13: a comparison between first-trimester combined screening and a cfDNA-based approach. Arch Gynecol Obstet. 2019;299:431-437. doi:10.1007/s00404-018-4983-2

21. Reiff ES, Little SE, Dobson L, et al. What is the role of the 11- to 14-week ultrasound in women with negative cell-free DNA screening for aneuploidy? Prenat Diagn. 2016;36:260-265. doi:10.1002/pd.4774

22. Mack LM, Lee W, Mastrobattista JM, et al. Are first trimester nuchal septations independent risk factors for chromosomal anomalies? J Ultrasound Med. 2017;36:155-161. doi:10.7863/ultra.16.01066

23. Mak A, Lee H, Poon CF, et al. Factors associated with common and atypical chromosome abnormalities after positive combined first-trimester screening in Chinese women: a retrospective cohort study. BMC Pregnancy Childbirth. 2019;19. doi:10.1186/s12884019-2205-y

24. Tang Y, Luo H, Mu D, et al.Early diagnosis of trisomy 21 , trisomy 18 and trisomy 13 using nuchal translucency thickness and ductus venosus blood flow waveform in West China. Mol Med Rep. 2018;19:1349-1355.

25. Tercanli S, Miny P, Filges I. Increased fetal nuchal translucency - also a risk for a rare submicroscopic chromosomal abnormalities. Ultraschall in Der Medizin. 2015;36:419-420.

26. Socolov D, Socolov R, Gorduza VE, et al. Increased nuchal translucency in fetuses with a normal karyotype - diagnosis and management: an observational study. Medicine. 2017;96:e7521. doi:10.1097/ MD.0000000000007521

27. Le Lous M, Bouhanna P, Colmant C, et al. The performance of an intermediate 16th-week ultrasound scan for the follow-up of euploid fetuses with increased nuchal translucency. Prenat Diagn. 2016;36:148-153. doi:10.1002/pd.4756

28. Kane SC, Reidy KL, Norris F, et al. Chorionic villus sampling in the cell-free DNA aneuploidy screening era: careful selection criteria can maximise the clinical utility of screening and invasive testing. Prenat Diagn. 2017;37:399-408. doi:10.1002/pd.5026

29. Lithner CU, Kublickas M, Ek S. Pregnancy outcome for fetuses with increased nuchal translucency but normal Karyotype. J Med Screen. 2015;23:1-6.

30. Ztürk FH, Cal FD, Erol SA, et al. Fetal genetic diagnosis by chorionic villus sampling: evaluation of the five-year experience from a single center. Fetal Pediatr Pathol. 2020;3:1-9. 


\section{Publish your work in this journal}

The International Journal of General Medicine is an international, peer-reviewed open-access journal that focuses on general and internal medicine, pathogenesis, epidemiology, diagnosis, monitoring and treatment protocols. The journal is characterized by the rapid reporting of reviews, original research and clinical studie across all disease areas. The manuscript management system is completely online and includes a very quick and fair peer-review system, which is all easy to use. Visit http://www.dovepress.com/ testimonials.php to read real quotes from published authors.

Submit your manuscript here: https://www.dovepress.com/international-journal-of-general-medicine-journal 